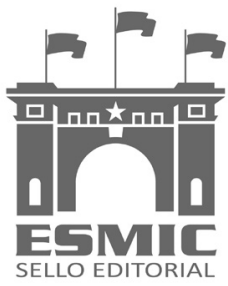

Revista Científica General José María Córdova

ISSN 1900-6586 (impreso), 2500-7645 (en línea)

Volumen 16, Número 24, octubre-diciembre 2018, pp. VII-X

http://dx.doi.org/10.21830/19006586.415

Citación: Fernandez-Osorio, A. \& Suarez Pineda, J. (2018, octubre-diciembre). Editorial: Agradecimientos a los pares evaluadores de la Revista Científica General José María Córdova del año 2018. Revista Científica General José María Córdova, 16 (24), vII-x DOI: http://dx.doi.org/10.21830/19006586.415

\title{
Editorial: Agradecimientos a los pares evaluadores de la Revista Científica General José María Córdova del año 2018
}

\author{
Andrés Eduardo Fernández-Osorio ${ }^{a}$ \\ Universidad de Barcelona, España \\ Jesús Alberto Suárez Pineda ${ }^{\mathrm{b}}$ \\ Escuela Militar de Cadetes "General José María Córdova”, Bogotá, Colombia
}

Editorial: Acknowledgments to the 2018 peer reviewers of the Revista Científica General José María Córdova

Editorial: Agradecimentos aos revisores da Revista Científica General José María Córdova do ano de 2018

Éditorial : Remerciements aux pairs examinateurs du La Revista Científica General José María Córdova de l'année 2018

a https://orcid.org/0000-0003-0643-0258 - Contacto: andres.fernandez@esmic.edu.co

b https://orcid.org/0000-0002-1155-3175 - Contacto: revistacientifica@esmic.edu.co 
La revisión por pares evaluadores es una parte fundamental del proceso de publicación de artículos de la Revista Científica General José María Córdova (Revista colombiana de estudios militares estratégicos), ya que avala la vigencia y calidad de sus contenidos.

Por tal motivo, la Revista publica, como justo reconocimiento, la relación de los académicos, docentes e investigadores, que participaron voluntariamente en la revisión de los artículos durante el año 2018; así como los miembros del comité editorial y comité científico que garantizaron la interdisciplinariedad y objetividad de los contenidos.

\section{Editores}

Andrés Eduardo Fernández-Osorio

Universidad de Barcelona, España

Jesús Alberto Suárez Pineda

Escuela Militar de Cadetes "General José María Córdova”, Bogotá, Colombia

\section{Comité editorial}

Rafael Martínez Martínez, PhD

Universidad de Barcelona

Barcelona, España

Maxim V. Bratersky, PhD

Escuela Superior de Economía

Moscú, Federación de Rusia

Fabricia Silva da Rosa, PhD

Universidad Federal de Santa Catarina

Florianópolis, Brasil

Alessandra Ciurlo, PhD

Pontificia Universidad Gregoriana

Roma, Italia

Daniel Legacé-Roy, PhD

Real Colegio Militar del Canadá

Kingston, Canadá.

Philippe Dufort, PhD

Universidad de Saint Paul

Ottawa, Canadá

Eutimio Mejía Soto, $\mathrm{PhD}(\mathrm{c})$

Universidad del Quindío

Armenia, Colombia 


\section{Comité Científico}

Luis Alfonso Ramírez Peña, PhD

Universidad Pedagógica Nacional

Bogotá, Colombia

Salvador Ceja Oseguera, $\mathrm{PhD}$

Universidad Popular Autónoma del Estado de Puebla

Puebla, México

Jaime Humberto Leiva de Antonio, PhD

Universidad del Valle

Cali, Colombia

Víctor Rafael Martin Fiorino, PhD

Universidad Católica de Colombia

Bogotá, Colombia

Vicente Torrijos Rivera, PhD(c)

Universidad del Rosario

Bogotá, Colombia

\section{Pares evaluadores}

Adriana Otálora Buitrago

Alejandro Ortiz Ríos

Alfredo Hernández Arciniegas

Álvaro Carrizosa de la Torre

Andrés Fernández Osorio

Andrés Felipe Cardona Orozco

Boris Alberto Pinzón Franco

Carlos Romero Huertas

Carlos Alberto Ardila Castro

Carlos Enrique Álvarez Calderón

Cecilia Garzón Daza

Cesar Castañeda Plata

César Yamit Beltrán Torres

Claudia Garay Acevedo

Daniel Lagacé-Roy

Diego Alexander Tibaduiza Burgos

Dustin Gómez Rodríguez

Edgar García López

Gustavo Andrés Cañas Peña

Henry Romero León 
Jaime Alfonso Cubides Cárdenas

Jaime Humberto Leiva Deantonio

Jennie Carignan

Jenny Alexandra Fuentes Quintero

Jesús Suarez Pineda

Jesús Eduardo Moreno Peláez

Jhon Jairo Losada Cubillos

Jonnathan Jiménez Reina

Juan Antonio Contreras Montes

Juan Carlos Ramos Mallarino

Julián Vargas Cardona

Laura Lucía Amézquita García

Leidy Johana Cabrera Cabrera

Lucia Meneses Lucumi

Luis Alexander Montero Moncada

Luis Antonio González Santos

Luis Horacio Pulgarín Montoya

Luz Estela Valencia Ayala

Luz Yolanda Morales Martín

Marcos Fortes

María Antonieta Corcione Nieto

Miguel González Martínez

Minerva Campion Canelas

Nora Patricia Gutiérrez Rodríguez

Nubia Leonor Posada González

Omar Vivas Cortes

Oscar Barrera Herrera

Paola Alexandra Sierra Zamora

Patricia Barrero de Rivera

Paula Janyn Melo Buitrago

Pedro Buitrago Rincón

Rafael Franco Ruíz

Ricardo Esquivel Triana

Salvador Ceja Oseguera

Samuel Mutarindwa

Samuel Kamugisha

Tainá Ribas Mélo

Valerio Nepomuceno

Wisman Johan Díaz Castillo

Yuliana Gómez Zapata 